\title{
BioFuelDB: a database and prediction server of enzymes involved in biofuels production
}

\author{
Nikhil Chaudhary ${ }^{1}{ }^{\text {, Ankit Gupta }}{ }^{1}$, Sudheer Gupta ${ }^{1}$, Vineet K Sharma ${ }^{\text {Corresp. }}{ }^{1}$ \\ ${ }^{1}$ Department of Biological Sciences, Indian Institute of Science Education and Research, Bhopal, Madhya Pradesh, INDIA \\ Corresponding Author: Vineet K Sharma \\ Email address: vineetks@iiserb.ac.in
}

Background. The rapid decrease in fossils fuel reserves and increasing demand for energy, novel methods to explore alternative biofuel production processes to alleviate these pressures. A wide variety of molecules which can either be used as biofuels or as biofuel precursors are produced using microbial enzymes. However, the common challenges in the industrial implementation of enzyme catalysis for biofuel production are the unavailability of a comprehensive biofuel enzyme resource, low efficiency of known enzymes, and limited availability of enzymes which can function under extreme conditions in the industrial processes.

Methods. We have developed a comprehensive database of known enzymes with proven or potential applications in biofuel production through text mining of PubMed abstracts and other publicly available information. A total of 131 enzymes with a role in biofuel production were identified and classified into six enzyme classes and four broad application categories namely 'Alcohol production', 'Biodiesel production', 'Fuel Cell' and 'Alternate biofuels'. A prediction tool 'Benz' was developed to identify and classify novel homologues of the known biofuel enzyme sequences from sequenced genomes and metagenomes. 'Benz' employs a hybrid approach incorporating HMMER 3.0 and RAPSearch2 programs to provide high accuracy and high speed for prediction.

Results. Using the Benz tool, 153,754 novel homologues of biofuel enzymes were identified from 23 diverse metagenomic sources. The comprehensive data of curated biofuel enzymes, their novel homologs identified from diverse metagenomes, and the hybrid prediction tool Benz are presented as a web server which can be used for the prediction of biofuel enzymes from genomic and metagenomic datasets. The database and the Benz tool is publicly available at $\mathrm{http}$ ://metabiosys.iiserb.ac.in/biofueldb $\&$ http://metagenomics.iiserb.ac.in/biofueldb . 
1 Title - BioFuelDB: a database and prediction server of enzymes involved in biofuels production

2 Authors

3 Nikhil Chaudhary*, Ankit Gupta*, Sudheer Gupta, Vineet K. Sharma $†$

\section{Affiliation}

5 Department of Biological Sciences, Indian Institute of Science Education and Research, Bhopal,

6 Madhya Pradesh, India

\section{7 † Corresponding Author}

8 Dr. Vineet K. Sharma, vineetks@iiserb.ac.in

$9 \quad *$ These authors contributed equally to this work 


\section{ABSTRACT}

Background. The rapid decrease in fossils fuel reserves and increasing demand for energy, novel methods to explore alternative biofuel production processes to alleviate these pressures. A wide variety of molecules which can either be used as biofuels or as biofuel precursors are produced using microbial enzymes. However, the common challenges in the industrial implementation of enzyme catalysis for biofuel production are the unavailability of a comprehensive biofuel enzyme resource, low efficiency of known enzymes, and limited availability of enzymes which can function under extreme conditions in the industrial processes.

Methods. We have developed a comprehensive database of known enzymes with proven or potential applications in biofuel production through text mining of PubMed abstracts and other publicly available information. A total of 131 enzymes with a role in biofuel production were identified and classified into six enzyme classes and four broad application categories namely 'Alcohol production', 'Biodiesel production', 'Fuel Cell' and 'Alternate biofuels'. A prediction tool 'Benz' was developed to identify and classify novel homologues of the known biofuel enzyme sequences from sequenced genomes and metagenomes. 'Benz' employs a hybrid approach incorporating HMMER 3.0 and RAPSearch2 programs to provide high accuracy and high speed for prediction.

Results. Using the Benz tool, 153,754 novel homologues of biofuel enzymes were identified from 23 diverse metagenomic sources. The comprehensive data of curated biofuel enzymes, their novel homologs identified from diverse metagenomes, and the hybrid prediction tool Benz are presented as a web server which can be used for the prediction of biofuel enzymes from genomic and metagenomic datasets. The database and the Benz tool is publicly available at $\underline{\text { http://metabiosys.iiserb.ac.in/biofueldb } \& \text { http://metagenomics.iiserb.ac.in/biofueldb }}$ 


\section{INTRODUCTION}

The global increase in energy demand and decline in the available stock of fossil fuels has become a challenge and requires a search for alternate sources of fuels and energy. In this scenario, enzyme catalyzed conversion of biomass to biofuels provides an ideal source of clean, ecological friendly and sustainable energy (Fortman et al. 2008). Bioalcohol and Biodiesel are the two major commercial biofuels in use today. Bioalcohols including methanol, ethanol and higher alcohols such as butanol, can be produced through microbial fermentation of sugar (first generation biomass) or lignocellulosic biomass (second generation biomass) and can be directly used as fuel (Canilha et al. 2012). In contrast, biodiesel refers to structurally tailored fatty esters which can be used as a replacement for traditional petroleum fuels (Steen et al. 2010). Biodiesel can be produced from edible and non-edible oils such as animal fats, cooking oil, algae oil and microbial lipids, through a trans-esterification process (Wang et al. 2015). Although the demand for biodiesel is increasing, the use of biodiesel has a disadvantage of increasing the prices and reducing the availability of vegetable crops for meeting the global food demand. Hence, in this scenario, microbial fermentation of biomass (including non-food crops) for the production of biodiesel serves as an ideal alternative medium (Oyola-Robles et al. 2014). The common strategies involving microbial fermentation for biodiesel production include enhancing the expression of enzymes involved in the production of biodiesel precursor, such as fatty acids and triglycerides, and deletion of genes involved in the fatty acid degradation pathways (OyolaRobles et al. 2014; Tamano et al. 2013).

The third major category of biofuels includes the microbial and enzymatic fuel cells. Microbial fuel cells are devices where microbes grow on the organic content and generate electric current. However, enzymatic fuel cells utilize cell free enzymes as electrodes for achieving the same functionality. In the previous studies, enzymatic fuel cells have been constructed using enzymemediated redox reactions at either or both of the electrodes (Kakehi et al. 2007; Yuhashi et al. 2005). In addition to these three biofuels, other alternatives such as terpenes, fatty aldehydes, fatty alcohols, biogas, etc, have been explored with varied success (Kung et al. 2014; Lennen \& Pfleger 2013; Zieminski et al. 2012).

Despite the merits of producing biofuels using enzymes, only a limited number of enzymes have been commercially exploited. It is primarily because of the unavailability of efficient enzymes 
63 that can perform the desired conversion and their inability to adapt to the application conditions 64 which may not be optimum for a given enzyme, hence leading to a decrease in efficiency (Bhardwaj Ajay et al. 2015). Consequently, the total share of all biofuels is 4\% of global road transport fuel demand because of the higher cost of production compared to gasoline, low quality and uneven composition of the end products, low efficiency of biofuel enzymes, and loss of functionality of enzymes in adverse $\mathrm{pH} /$ temperature/chemical composition of the culture medium of the reactors (Bhardwaj Ajay et al. 2015).

Therefore, to look for efficient and novel variants of enzymes involved in the different steps of biofuel production (referred to as 'biofuel enzymes' in the subsequent text), the first task is to expand our knowledgebase by exploring the naturally occurring biofuel enzymes and to search for homologues of these enzymes from natural environments. The application of high-throughput sequencing technologies has revealed the sequences of thousands of genomes which promise to facilitate the discovery and identification of novel biofuel enzymes. Furthermore, metagenomics has been developed into a culture independent approach that explores the diversity and complexity of microbial genomes in their natural environments and provides the information on novel genes and pathways from yet unculturable genomes. Thus, genome sequencing and metagenomics would assist in increasing the enzyme repertoire by revealing novel biofuel enzymes, and can also provide us with the functional variants of the existing enzymes. The availability of multiple metagenomic databases provides a useful opportunity to discover novel homologs of existing biofuel enzymes.

Several studies and databases have reported enzymes that can be used for biofuel production (Choi et al. 2013; Lombard et al. 2014; Misra et al. 2016; Yin et al. 2012). For example, the database of enzymes of microbial biofuel feedstock (dEMBF) provides information related to algal biofuel research but is limited to only 15 sequenced microalgal species and their implication in lipid synthesis (Misra et al. 2016). At present, there is no comprehensive database which is dedicated for retrieving information on biofuel enzymes. Hence, in this work, we have developed BioFuelDB, a comprehensive database of enzymes with demonstrated or potential application(s) in biofuel production by searching the available scientific literature. Using this database, a prediction tool 'Benz' was constructed by exploiting the homology-based and profile-based approaches to search for the potential homologs of existing biofuel enzymes. 
93 BiofuelDB is freely available at http://metabiosys.iiserb.ac.in/biofueldb and 94 http://metagenomics.iiserb.ac.in/biofueldb.

95

96

97

98 99

100

101

102

103

104

105

106

107

108

109

110

111

112

113

114

115

116

117

118

119

120

121

\section{METHODS}

\section{Construction of BioFuelDB}

The flowchart of the methodology used for the construction of BioFuelDB is shown in Figure 1.

\section{List of curated biofuel enzymes}

The initial database of enzymes was constructed by searching the available 'English' abstracts containing the terms 'biofuel AND enzyme', 'biodiesel AND enzyme', 'alcohol AND enzyme', 'ethanol AND enzyme', 'methanol AND enzyme', 'fuel cell AND enzyme' and 'alternate biofuel' at NCBI PubMed and were imported to into a MySQL Database version 14.14 (Coordinators 2013). The initial set of candidate enzymes were identified using the 'Natural Language full-text search' and 'Boolean full-text search' features of MySQL. From this initial set of 2,562 abstracts, enzymes having demonstrated applications in the production of any category of biofuel(s) were manually curated. For the resultant 131 enzymes, the Enzyme Commission (EC) numbers were used as the unique identifiers to refer to individual enzymes in the BioFuelDB database. Based on their known application, the enzymes were classified into four broad categories namely 'Alcohol production', 'Biodiesel production', 'Fuel Cell' and 'Alternate biofuels'. An enzyme could be classified into more than one application category based on its known applications. All enzymes which are known to be involved in different steps of biofuel production are referred to as 'biofuel enzymes' in this manuscript.

\section{Enzyme Sequence Collection}

Protein sequences for the curated list of enzymes were obtained from SwissProt database for all 131 enzymes. Sequences marked as 'putative', 'probable' or 'hypothetical' were removed from this initial set of sequences. The enzymes for which no SwissProt sequences remained after the removal of such sequences were discarded. SwissProt, which is a curated protein sequence database and provides high-quality annotation, was the preferred source for extracting the sequences over TrEMBL, which is a computer-annotated supplement to Swiss-Prot. However, for enzymes with less than five sequences available in the SwissProt database, sequences from 
122 TrEMBL were included. After following the above two steps, all enzymes with at least five

123 representative sequences were included, and this database was termed as the Primary database.

124 This primary database consisted of 8,263 sequences representing 131 selected enzymes.

\section{Other Resources}

126 Information about the reaction(s) catalyzed by the enzyme, its substrate(s), product(s), KEGG

127 Orthology, and KEGG Pathways was obtained from the KEGG database (Kanehisa et al. 2014).

\section{Construction of 'Benz'}

129 The Benz tool was developed to identify novel homologues of the known biofuel enzyme 130 sequences from sequenced genomes and metagenomes. This tool employs a hybrid approach 131 incorporating HMMER 3.0 and RAPSearch2 programs to provide high accuracy and high speed 132 for prediction (Eddy 2008; Zhao et al. 2012). It is a useful strategy to adopt a hybrid approach 133 involving two different methods for predicting the novel homologs. The homology-based approach using RAPSearch2 would enable the direct detection of close homologs of the known biofuel enzymes. However, to overcome the limitations of homology-based pairwise alignment in detecting remote homologs, profile-based methods implemented using HMMER 3.0 help to seek distant evolutionary relationships to detect remote homologs. Profile-based methods such as HMMER, consider the information of evolutionarily related sequences derived from multiple sequence alignments and gains sensitivity by including position-specific information into an alignment process by computing variation across related sequences at each position. Thus, HMMER3 predictions can be utilized by the user to detect more variant enzymes. To implement the HMM module, the protein sequences of the enzymes from the four categories were searched against the Pfam domains database. In total, 336 domains matched the sequences of the total set of 131 enzymes. However, several of these Pfam domains represent general functions (e.g., ATB binding domain, DNA binding domain, various loops etc) and could not represent the specific function of the enzyme. Because of the lack of sufficient and conclusive information available

147 through Pfam domains, HMM profiles were constructed for each enzyme. All sequences belonging to an enzyme were grouped together and aligned using the 'ClustalW' program (Larkin et al. 2007). The resulting alignment files were used as the input for 'hmmbuild' function of the HMMER 3.0 program to build HMM profiles. By combining HMM profiles of all 131 individual enzymes, a small HMM profile database was constructed exclusively for biofuels 
152 'BioFuel-Pfam', which was further used as a reference database to identify homologs of the 153 existing biofuel enzymes. As a parallel approach, the homology-based method 'RAPSearch2' 154 along with profile-based 'HMMER3.0' was implemented to attain higher confidence in 155 predictions using two different methods. The sequences representing individual enzymes as well 156 as the different categories were processed using the 'prerapsearch' function of the RAPSearch2 157 program to build 'RAPSearch database' and were directly included in the Benz program. The 158 two databases 'Biofuel-PfamDB' and 'RapsearchDB', constructed in the above sections were 159 used as reference databases for the query sequences.

160

161

162

163

164

165

166

167

\section{Test Datasets for the Evaluation of Benz Efficiency}

Two test datasets were constructed to evaluate the performance of Benz program. In the first test dataset, a database of test sequences was prepared from the 'hypothetical', 'probable' and 'putative' sequences which were discarded during the preparation of the Primary database. This dataset consisted of 25,630 protein sequences. The results of the Benz output were compared with the known annotation of the sequences as well as with the results of the BLAST (blastp program with evalue cutoff 1e-6). To construct the second test dataset, ORFs from three different metagenomes (MG-RAST ids: mgm4466309, mgm4516289 and mgm4559623) were downloaded from the MG-RAST web server. A total of 549,870 ORF sequences from the three metagenomes were analyzed using Benz, and the results were compared using a BLAST search. The following standard parameters were used for accessing the efficiency of the program.

$$
\begin{gathered}
\text { Sensitivity }=\frac{t p}{t p+f n} \times 100 \\
\text { Specificity }=\frac{t n}{t n+f p} \times 100 \\
\text { Accuracy }=\frac{t p+t n}{t p+f n+t n+f p} \times 100 \\
\text { Matthews Correlation Coefficient }(M C C)=\frac{(t p)(t n)-(f p)(f n)}{\sqrt{(t p+f p)(t p+f n)(t n+f p)(t n+f n)}}
\end{gathered}
$$

where 'tp' (true positives) are the sequences which belong to a given class of BioFuelDB enzymes and have been assigned to the same class by Benz, 'tn' (true negatives) are the 
178 sequences which do not belong to a given class of BioFuelDB enzymes and have not been 179 assigned, 'fp' (false positives) are the sequences which do not belong to a given class but have 180 been incorrectly assigned to it, and 'fn' (false negatives) are the sequences which belong to a 181 given class but have been incorrectly assigned to some other class. Only the hits with the tag ' $\mathrm{C}$ ' 182 were considered as acceptable results from Benz and were used for the performance assessment 183 of the tool.

184 Metagenomic Datasets for Mining Biofuel Enzymes

185 A total of 23 metagenomic datasets consisting of 22,470,288 ORFs were downloaded from MG186 RAST and analyzed using "Benz" for the discovery of biofuel enzymes. These 23 selected 187 metagenomes include sequences from diverse environments including marine, extreme saline, 188 fresh water, aquatic, grasslands, hot springs, coral reef, extreme aquatic habitat (drilling), forests, 189 village, activated sludge, hydrothermal vents, lakes, anthropogenic terrestrial biome and 190 cropland.

191

192

193

\section{RESULTS AND DESCRIPTION OF THE WEB SERVER}

\section{Distribution of Enzymes Across Application Categories}

The distribution of 131 enzymes in four application-based categories provides an overall summary of the availability of enzymes for the production of various types of biofuels classified in the four categories. Out of the four categories, 'Alcohol production' contains the highest (74), followed by Biodiesel (30), Fuel Cell (27) and 'Alternate' which contains the lowest (19) number of enzymes (Table 1 and Table S1). This implies that almost half of the total numbers of known biofuel enzymes are involved in alcohol production. As mentioned earlier, selected enzymes may be present in more than one category if they were found to perform reactions involved in multiple categories.

202

Analysis of enzyme distribution across various EC classes in Primary database reveals some 203 interesting observation. EC5 and EC6, representing 'Isomerases' and 'Ligases', contained least number of enzymes ( 3 and 4, respectively) in the database, whereas, the highest number of enzymes belonged to EC1 and EC3 classes, representing 'Oxidoreductases' and 'Hydrolases' (44 and 42, respectively) (Table 1 and Table S2). This can be ascribed to their high occurrences in 
208 production' the largest percentage of enzymes in the Primary database was in EC1 and EC2 209 (50.44\% and 20.99\%), as these categories correspond to oxidoreductases and hydrolases, 210 respectively. In case of 'Fuel Cells', majority of enzymes belonged to EC1 class, as 211 oxidoreductases make good candidates for application at electrodes. In the case of 'Biodiesel 212 production', the enzyme distribution was more widely spread across EC classes as biodiesel production occurs through a long chain of reactions employing a variety of enzymes.

\section{Performance Evaluation of Benz}

215 In case of the first test dataset, out of 24,009 sequences, Benz classified 23,014 sequences as 216 biofuel enzymes, of which 14,456 were classified as 'consensus' results. From these consensus 217 results, average accuracy values of $95.56 \%$ and $92.20 \%$ were observed for the enzyme classes 218 and application categories, respectively (Figure 2). RAPSearch2 was able to classify 22,196 219 sequences with an average accuracy of $93.89 \%$ and $94.68 \%$ for the enzyme classes and application categories, respectively (Figure 2). Whereas, HMMER3 could classify 22,388 sequences with an average accuracy of $59.14 \%$ and $54.07 \%$ for various EC classes and application categories, respectively (Figure 2). The other parameters of performance evaluation of test dataset 1 are shown in supplementary tables S3 and S4.

In case of dataset 2, the predictions of Benz, RAPSearch and HMMER were compared with the results from BLAST search since the annotations of the sequences of this dataset were unknown. Out of 549,870 sequences, BLAST classified 16,678 sequences as biofuel enzymes, whereas Benz classified 23,317 as biofuel enzymes. As the BLAST annotation was available only for 16,678 sequences, the performance measurement of Benz was performed only on these set of sequences, and the other predictions made by Benz could not be evaluated. Benz classified 7,292 sequences as 'consensus' sequences with the average accuracy of $98.64 \%$ and $97.89 \%$, respectively, for various EC classes and application categories (Figure 2). Using only RAPSearch2, 13,468 sequences could be classified with an average accuracy of $95.68 \%$ and 93.48\%, respectively, for various EC classes and application categories (Figure 2). Similarly, using only HMMER3, 21,177 sequences could be classified with an average accuracy of 73.09\% and $66.79 \%$, respectively, for various EC classes and application categories (Figure 2). The other parameters of performance evaluation of test dataset 2 are shown in supplementary tables S5 and S6. 
238 As evident from the performance evaluation of Benz on test datasets 1 and 2, the consensus

239 results are reliable with high sensitivity, specificity, accuracy, and MCC values, although this

240 performance comes at the cost of percentage prediction. Rapsearch2 also provides a good

241 performance while maintaining a high percentage prediction value. HMMER3, however,

242 displays a variable performance on different datasets as well as across different application

243 categories and EC classes. Thus, 'consensus' results can be considered more reliable, whereas

244 HMMER3 predictions can be utilized by the user to detect more variant enzymes. Further, the

245 availability of a profile-based search provides additional options to the users to search for biofuel

246 enzymes using an alternative approach.

\section{Identification of Novel Homologues in Metagenomic Datasets}

248 A total of 23 metagenomes from a variety of biomes were downloaded from the MG-RAST 249 server [26]. Using the Benz program with stringent E-values of E-6 for RAPSearch2 and E-21 250 for HMMER, a total of 153,754 novel homologues could be identified from these metagenomes

251 (Table 2 and Table 3). The distribution of these enzymes predicted from different metagenomes 252 showed a similar distribution pattern as observed in the global distribution. Across all categories, 253 the highest $(85,138)$ number of enzymes were present in the Bioalcohol category, followed by 254 the Fuel Cells category (51,841). Biodiesel and Alternate Biofuels had 22,143 and 25,391 255 sequences, respectively, which were similar to the patterns observed earlier in the test dataset2 256 (Table 2 and Table 3). Across EC classes, EC1 and EC2 were the most abundant (57,398 and 25731,602 respectively), whereas EC5 contained the least number of sequences $(3,917)$.

258 From all considered metegenomes, around $0.5 \%$ to $1.5 \%$ of total ORFs were identified as 259 Biofuel enzymes, which is a significant indication of their prevalence. Cropland, forest and other 260 biomes (such as grassland and village biome) with degrading biomass were found to contain a 261 higher proportion (1.8\%) of enzymes under the 'Bioalcohol' category. This implies the presence 262 of a large number of alcohol producing (fermentative) enzymes in the microbiome of these 263 environments(Limayem \& Ricke 2012). Similarly, considerable proportions (0.31 to $1.81 \%)$ of

264 biofuel enzymes were found in aquatic, marine and extreme environments. Furthermore, 21,553 265 homologues were identified from 5 extreme habitats consisting of hypersaline, drilling and 266 hydrothermal vent and hot spring biomes (Table 2). The use of enzymes which can survive at 267 high temperatures (thermostable enzymes) enables the bioprocess temperatures higher than the 
268 distillation point of ethanol, in which case the gasified biofuels are collected from continuous

269 bioprocess preventing end product inhibition (Yeoman et al. 2010). Similarly, the enzymes

270 which can survive in hyper saline environments can catalyze the process of degrading cellulosic

271 biomass for biofuel production (Begemann et al. 2011). These analyses suggest that the available

272 metagenomic data from different environments provide possibilities of identifying novel

273 homologs of Biofuel enzymes which could be commercially exploited.

274 Description of BioFuelDB Web Server

275 The Primary database consisting of 8,236 sequences of biofuel enzymes and 153,754 sequence

276 homologs of biofuel enzyme (metalog), which were mined from the metagenomes, were

277 combined to form the BioFuelDB database which was incorporated in the Web Server.

\section{Explore Page}

279 The 'Explore' page of the web server allows the user to search the enzymes from the BioFuelDB 280 database by enzyme name, Enzyme Commission (EC) number, enzyme's systematic name or 281 enzyme's KEGG Reaction ID(s). The user can also browse the database by application category 282 of the enzymes, i.e., 'Alcohol Production', 'Biodiesel Production', 'Fuel Cell', 'Alternate Biofuels' 283 or all the enzymes irrespective of the application category. Selecting an enzyme name takes the 284 user to a new page where the various information about the enzyme are displayed such as, 285 systematic name, EC number, common name(s) of the enzyme, application category, chemical 286 reaction undertaken by the enzyme, KEGG reaction $\operatorname{ID}(\mathrm{s})$, substrates and products of the 287 enzyme's reaction, the biological pathways in which the enzyme is involved and KEGG 288 Orthology. Furthermore, the page provides the UniProt sequences of the enzyme in FASTA 289 format as well as the PubMed references on which the application of the enzyme in the given 290 application category was demonstrated.

\section{Prediction}

292 The 'Prediction' page of the BioFuelDB web server is designed to identify novel homologues of 293 the enzymes available in the BioFuelDB database. This page employs a hybrid tool 'Benz' consisting of 'Biofuel-PfamDB' and 'RapsearchDB' databases at the backend for the prediction of novel homologs of biofuel enzymes which can be used for the production of biofuels. The user can either provide raw FASTA sequence(s) of proteins or ORFs in the input box or upload a query FASTA file through the 'Upload' interface. The query can be made either against all 
298 enzymes in the database, enzymes from any one application category, or against any one selected 299 enzyme. For RAPSearch, the default e-value for the search is E-6, and for HMM it is E-21.

300 The output is reported in tab-separated format with nine columns namely: 'Query', 'HMM Hit' 301 (matching hit from the HMMER profile), 'HMM E-value', 'HMM Score', 'RAPSearch2 Hit' (hit 302 from the RAPSearch2 database), 'RAPSearch2 E-value', 'RAPSearch2 Score', 'Tags' 303 (comparison between the outputs from both modules), and 'Category' (application categories of 304 the predicted EC number). Tag ' $\mathrm{C}$ ' implies that the results from both the modules are in 305 consensus, ' $N$ ' implies that result from both the modules are not in consensus, ' $H$ ' implies that 306 only the HMMER module provided a result for the particular query, and similarly, ' $R$ ' implies 307 that only RAPSearch2 provided a result for the particular query. Queries with no hits from either 308 program are not reported in the results file.

\section{MetaLog Page}

310 The 'MetaLog' page provides links to download the metagenomic homologues of the 131 311 biofuel enzymes (length $>50$ amino acids) present in the Primary database enzymes predicted 312 from 23 varied metagenomes using the Benz tool. 


\section{DISCUSSION AND CONCLUSION}

314 The main motivation for this work was the unavailability of any specialized database which 315 provides comprehensive information on enzymes involved in different types of biofuel 316 production. The mining of literature revealed that only a limited number of enzymes involved in 317 biofuel production are currently known from a limited number of genomes. Therefore, as the first 318 step, we constructed the 'BioFuelDB' knowledgebase of all enzymes involved in biofuel 319 production from the available literature. However, the limited repertoire of these enzymes 320 becomes a limitation while selecting the enzyme variants which can perform the desired reaction 321 under the industrial conditions that may not be optimum for the given enzyme, hence leading to 322 decrease in efficiency (Bhardwaj Ajay et al. 2015). Thus, to explore efficient and novel variants 323 of enzymes involved in different steps of biofuel production, we have developed the Benz tool which can identify novel homologues of the known biofuel enzyme sequences from sequenced genomes and metagenomes. The hybrid approach incorporating HMMER 3.0 and RAPSearch2 programs provides high accuracy and high speed for prediction of biofuel enzymes. Further, it appears to be a useful strategy to adopt a hybrid approach involving two different methods as the homology-based method RAPSearch2 enables the identification of close homologs of the known biofuel enzymes, whereas, the profile-based method HMMER 3.0 helps to identify the remote homologs which show low sequence identity.

In the present scenario, metagenomic data generated from different environments comprising of sequences from culturable and unculturable microbial genomes can be mined to improve the repertoire of biofuel enzymes by revealing novel biofuel enzymes as well as the functional variants of the existing enzymes. In this study, the identification of 153,754 enzymes from 23 metagenomes indicates the possibility of finding such enzymes by exploiting the metagenomic data from several hundreds of metagenomes. Furthermore, the metagenomes are so rich in microbial diversity and functional genes that it is almost certain to identify the novel variant of a given enzyme (Sharma et al. 2010). Thus, the mining of novel homologues of biofuel enzymes from different environments using their metagenomic data enables the identification of novel variants which can work in wide range of conditions, and thus improves the enzyme repertoire.

To our knowledge, BioFuelDB is the first comprehensive dataset of biofuel enzymes. We anticipate that it would act as a comprehensive resource of biofuel enzymes and would assist 
343 researchers to explore novel variants of biofuel enzymes from different environments. However, 344 the efficiency of the novel variants can only be ascertained through laboratory experiments, but 345 the high quality of the initial primary database and stringent search criteria of Benz tool ensures 346 that all the predicted enzyme sequences can be used as leads for experimental validations. The 347 database and tools are available freely at the website http://metabiosys.iiserb.ac.in/biofueldb \& 348 http://metagenomics.iiserb.ac.in/biofueldb. 


\section{Acknowledgements}

350 Ankit Gupta is a recipient of DST-INSPIRE Fellowship and thanks the Department of Science 351 and Technology for the fellowship. 
352

353

354

355

356

357

358

359

360

361

362

363

364

365

366

367

368

369

370

371

372

373

374

375

376

377

378

379

380

381

382

383

384

385

386

387

388

389

390

391

392

393

394

395

396

397

\section{References}

Begemann MB, Mormile MR, Paul VG, and Vidt DJ. 2011. Potential Enhancement of Biofuel Production Through Enzymatic Biomass Degradation Activity and Biodiesel Production by Halophilic Microorganisms. In: Ventosa A, Oren A, and Ma Y, eds. Halophiles and Hypersaline Environments: Current Research and Future Trends. Berlin, Heidelberg: Springer Berlin Heidelberg, 341-357.

Bhardwaj Ajay K, Zenone T, and Chen J. 2015. Sustainable Biofuels, An Ecological Assessment of the Future Energy.

Canilha L, Chandel AK, Suzane dos Santos Milessi T, Antunes F, Luiz da Costa Freitas W, das Graças Almeida Felipe M, and da Silva S. 2012. Bioconversion of Sugarcane Biomass into Ethanol: An Overview about Composition, Pretreatment Methods, Detoxification of Hydrolysates, Enzymatic Saccharification, and Ethanol Fermentation. J Biomed Biotechnol 2012. 10.1155/2012/989572

Choi J, Kim KT, Jeon J, and Lee YH. 2013. Fungal plant cell wall-degrading enzyme database: a platform for comparative and evolutionary genomics in fungi and Oomycetes. BMC Genomics 14 Suppl 5:S7. 10.1186/1471-2164-14-S5-S7

Coordinators NR. 2013. Database resources of the National Center for Biotechnology Information. Nucleic Acids Res 41:D8-D20. 10.1093/nar/gks1189

Eddy SR. 2008. A probabilistic model of local sequence alignment that simplifies statistical significance estimation. PLoS Comput Biol 4:e1000069. 10.1371/journal.pcbi.1000069

Fortman J, Chhabra S, Mukhopadhyay A, Chou H, Lee TS, Steen E, and Keasling JD. 2008. Biofuel alternatives to ethanol: pumping the microbial well. Trends in biotechnology 26:375-381.

Kakehi N, Yamazaki T, Tsugawa W, and Sode K. 2007. A novel wireless glucose sensor employing direct electron transfer principle based enzyme fuel cell. Biosensors and Bioelectronics 22:2250-2255. http://dx.doi.org/10.1016/j.bios.2006.11.004

Kanehisa M, Goto S, Sato Y, Kawashima M, Furumichi M, and Tanabe M. 2014. Data, information, knowledge and principle: back to metabolism in KEGG. Nucleic Acids Res 42:D199-205. 10.1093/nar/gkt1076

Kung Y, McAndrew RP, Xie X, Liu CC, Pereira JH, Adams PD, and Keasling JD. 2014. Constructing tailored isoprenoid products by structure-guided modification of geranylgeranyl reductase. Structure 22:1028-1036. 10.1016/j.str.2014.05.007

Larkin MA, Blackshields G, Brown NP, Chenna R, McGettigan PA, McWilliam H, Valentin F, Wallace IM, Wilm A, Lopez R, Thompson JD, Gibson TJ, and Higgins DG. 2007. Clustal W and Clustal X version 2.0. Bioinformatics 23:2947-2948. 10.1093/bioinformatics/btm404

Lennen RM, and Pfleger BF. 2013. Microbial production of fatty acid-derived fuels and chemicals. Curr Opin Biotechnol 24:1044-1053. 10.1016/j.copbio.2013.02.028

Limayem A, and Ricke SC. 2012. Lignocellulosic biomass for bioethanol production: current perspectives, potential issues and future prospects. Progress in Energy and Combustion Science 38:449-467.

Lombard V, Golaconda Ramulu H, Drula E, Coutinho PM, and Henrissat B. 2014. The carbohydrate-active enzymes database (CAZy) in 2013. Nucleic Acids Res 42:D490-495. 10.1093/nar/gkt1178

Misra N, Panda PK, Parida BK, and Mishra BK. 2016. dEMBF: A Comprehensive Database of Enzymes of Microalgal Biofuel Feedstock. PLoS One 11:e0146158. 10.1371/journal.pone.0146158

Oyola-Robles D, Rullan-Lind C, Carballeira NM, and Baerga-Ortiz A. 2014. Expression of dehydratase domains from a polyunsaturated fatty acid synthase increases the production of fatty acids in Escherichia coli. Enzyme Microb Technol 55:133-139. 10.1016/j.enzmictec.2013.10.010

Sharma VK, Kumar N, Prakash T, and Taylor TD. 2010. MetaBioME: a database to explore commercially useful enzymes in metagenomic datasets. Nucleic Acids Res 38:D468-472. 10.1093/nar/gkp1001 
Steen EJ, Kang Y, Bokinsky G, Hu Z, Schirmer A, McClure A, Del Cardayre SB, and Keasling JD. 2010. Microbial production of fatty-acid-derived fuels and chemicals from plant biomass. Nature 463:559-562.

Tamano K, Bruno KS, Karagiosis SA, Culley DE, Deng S, Collett JR, Umemura M, Koike H, Baker SE, and Machida M. 2013. Increased production of fatty acids and triglycerides in Aspergillus oryzae by enhancing expressions of fatty acid synthesis-related genes. Applied microbiology and biotechnology 97:269-281. 10.1007/s00253-012-4193-y

Wang Y-T, Fang Z, Zhang F, and Xue B-J. 2015. One-step production of biodiesel from oils with high acid value by activated $\mathrm{Mg}-\mathrm{Al}$ hydrotalcite nanoparticles. Bioresource technology 193:84-89. http://dx.doi.org/10.1016/j.biortech.2015.06.059

Yeoman CJ, Han Y, Dodd D, Schroeder CM, Mackie RI, and Cann IK. 2010. Thermostable enzymes as biocatalysts in the biofuel industry. Adv Appl Microbiol 70:1-55. 10.1016/s0065-2164(10)700010

Yin Y, Mao X, Yang J, Chen X, Mao F, and Xu Y. 2012. dbCAN: a web resource for automated carbohydrate-active enzyme annotation. Nucleic Acids Res 40:W445-451. 10.1093/nar/gks479

Yuhashi N, Tomiyama M, Okuda J, Igarashi S, Ikebukuro K, and Sode K. 2005. Development of a novel glucose enzyme fuel cell system employing protein engineered PQQ glucose dehydrogenase. Biosensors and Bioelectronics 20:2145-2150. http://dx.doi.org/10.1016/j.bios.2004.08.017

Zhao Y, Tang H, and Ye Y. 2012. RAPSearch2: a fast and memory-efficient protein similarity search tool for next-generation sequencing data. Bioinformatics 28:125-126. 10.1093/bioinformatics/btr595

Zieminski K, Romanowska I, and Kowalska M. 2012. Enzymatic pretreatment of lignocellulosic wastes to improve biogas production. Waste Manag 32:1131-1137. 10.1016/j.wasman.2012.01.016 
Figure 1

Flowchart of the strategy used for constructing BioFuelDB

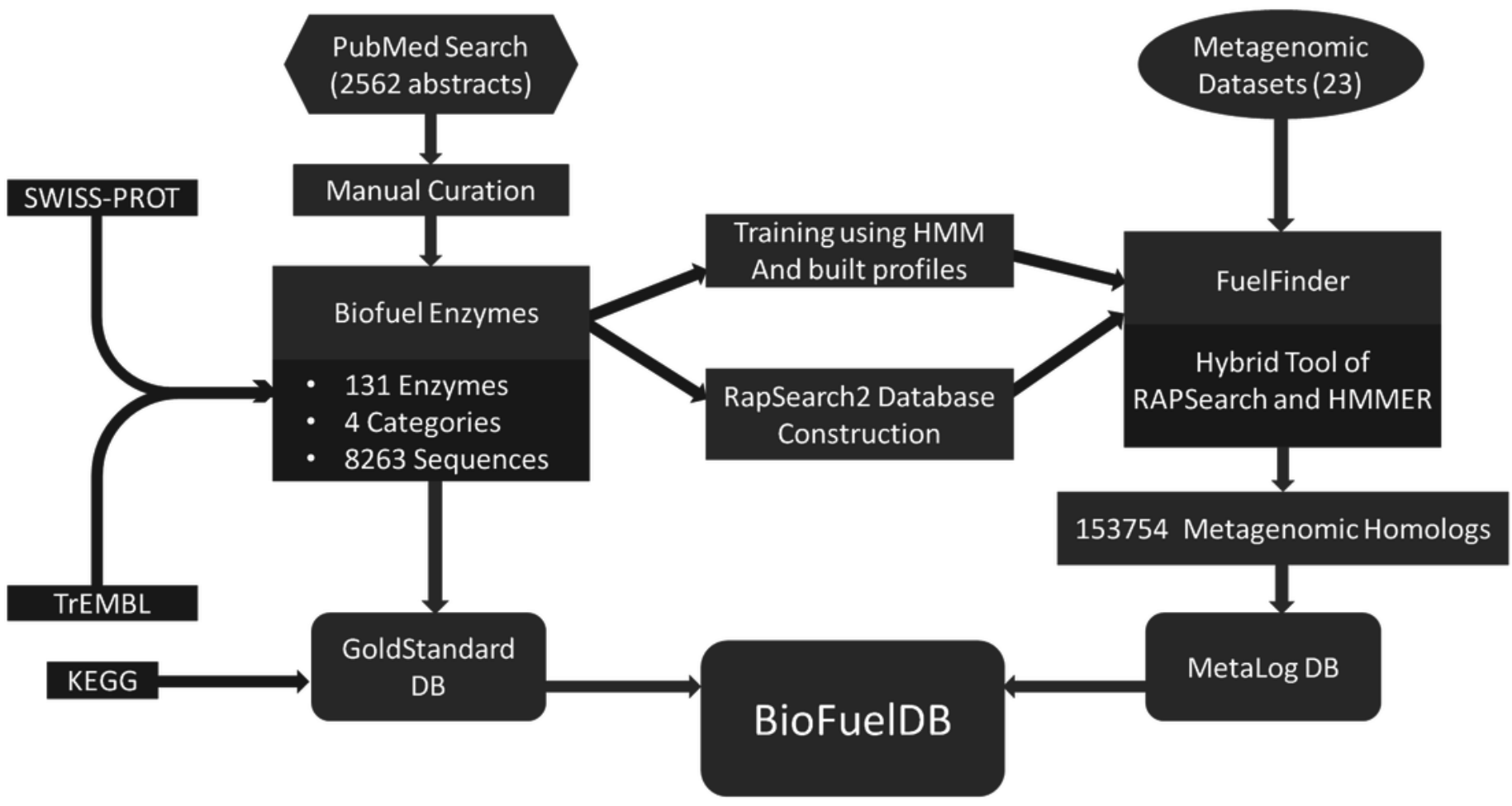




\section{Figure 2}

Performance comparison of HMMER, RAPSearch and Benz tool in terms of percentage prediction, accuracy for EC Classes and accuracy for application categories

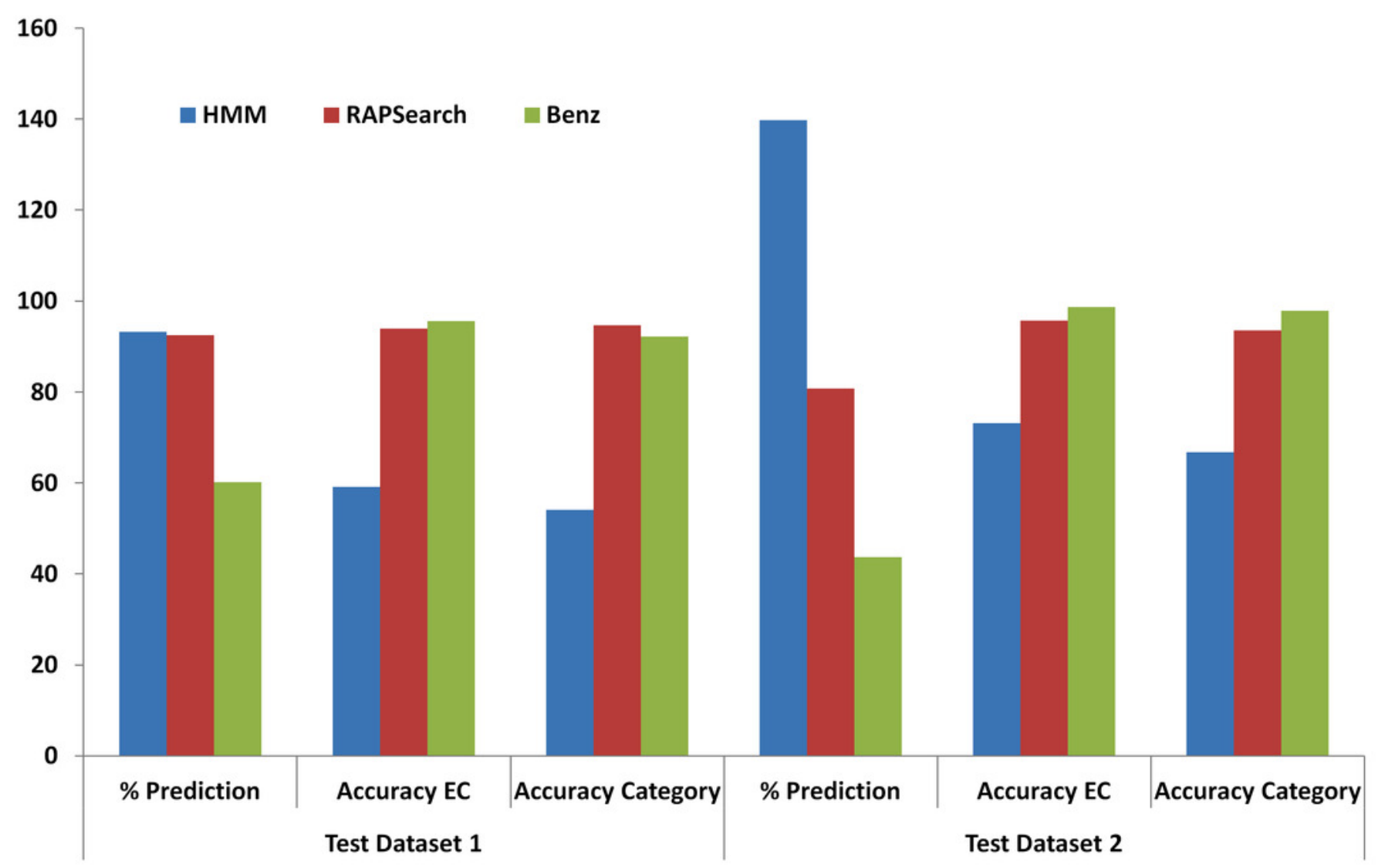




\section{Table $\mathbf{1}$ (on next page)}

Distribution of biofuel enzymes in four application categories and six EC classes 
1 Table 1: Distribution of biofuel enzymes in four application categories and six EC classes.

2

\begin{tabular}{|c|c|c|c|c|c|c|c|}
\hline & \multicolumn{7}{|c|}{ Number of Enzymes } \\
\hline Application Category & EC1 & EC2 & EC3 & EC4 & EC5 & EC6 & Total \\
\hline Alcohol Production & 20 & 8 & 33 & 10 & 2 & 1 & 74 \\
\hline Biodiesel & 4 & 13 & 7 & 4 & 0 & 2 & 30 \\
\hline Fuel Cell & 19 & 0 & 3 & 2 & 1 & 2 & 27 \\
\hline Alternate Biofuels & 5 & 7 & 4 & 3 & 0 & 0 & 19 \\
\hline
\end{tabular}

3 


\section{Table 2 (on next page)}

Distribution of novel homologs of biofuel enzymes (metalogs) in different application categories and metagenomes. 
1 Table 2. Distribution of novel homologs of biofuel enzymes (metalogs) in different application categories and metagenomes.

\begin{tabular}{|c|c|c|c|c|c|c|c|}
\hline Metagenome ID & Metagenome Source & $\begin{array}{l}\text { Total } \\
\text { ORFs }\end{array}$ & Alcohol & Biodiesel & Fuel Cell & Others & $\begin{array}{c}\text { Total } \\
\text { Biofuels }\end{array}$ \\
\hline mgm4440324 & Marine Biome & 36701 & 88 & 22 & 54 & 23 & 153 \\
\hline mgm4440329 & Hypersaline & 150513 & 175 & 37 & 93 & 36 & 278 \\
\hline mgm4441050 & Hypersaline & 3517 & 18 & 6 & 23 & 3 & 44 \\
\hline mgm4441102 & Hydrothermal Vent & 368502 & 2316 & 742 & 2070 & 1118 & 5231 \\
\hline mgm4443684 & Freshwater & 388210 & 1594 & 497 & 1430 & 568 & 3394 \\
\hline mgm4448052 & Aquatic Biome & 414473 & 1995 & 654 & 1674 & 920 & 4401 \\
\hline mgm4449252 & Grassland & 78039 & 801 & 224 & 475 & 261 & 1433 \\
\hline mgm4460449 & Hot Spring & 762819 & 5456 & 1928 & 4388 & 2419 & 11972 \\
\hline mgm4466309 & Coral Reef & 176426 & 1414 & 493 & 1041 & 457 & 2922 \\
\hline mgm4467029 & Large Lake & 376200 & 2029 & 810 & 1155 & 1836 & 5012 \\
\hline mgm4477803 & Lake & 5383950 & 9814 & 2911 & 7907 & 2706 & 19965 \\
\hline mgm4478241 & Extreme Aquatic Habitat (Drilling) & 222722 & 2702 & 417 & 1597 & 736 & 4028 \\
\hline mgm4479942 & Village Biome & 83867 & 761 & 216 & 529 & 274 & 1455 \\
\hline mgm4487639 & Forest Biome & 457998 & 882 & 215 & 451 & 152 & 1455 \\
\hline mgm4494621 & Activated Sludge & 5054731 & 14444 & 4727 & 12546 & 3063 & 28882 \\
\hline mgm4516289 & Aquatic Biome & 253233 & 1490 & 526 & 1010 & 674 & 3085 \\
\hline mgm4523306 & Anthropogenic Terrestrial Biome & 3007 & 18 & 2 & 5 & 1 & 24 \\
\hline mgm4527699 & Cropland Biome & 755188 & 7616 & 1160 & 2573 & 2149 & 11336 \\
\hline mgm4528623 & Cropland Biome & 238739 & 871 & 275 & 658 & 376 & 1860 \\
\hline mgm4537095 & Mediterranean Forests, Woodlands, Shrub & 360982 & 1653 & 541 & 824 & 415 & 2629 \\
\hline mgm4559623 & Aquatic Biome & 120211 & 771 & 155 & 401 & 291 & 1285 \\
\hline mgm4571849 & Aquatic Biome & 4464190 & 16310 & 3234 & 6262 & 4120 & 24525 \\
\hline mgm4571867 & Aquatic Biome & 2316070 & 11920 & 2351 & 4675 & 2793 & 18385 \\
\hline & Total & 22470288 & 85138 & 22143 & 51841 & 25391 & 153754 \\
\hline
\end{tabular}

2 


\section{Table 3(on next page)}

Distribution of novel homologs of biofuel enzymes (metalogs) in different EC Classes and metagenomes. 
1 Table 3. Distribution of novel homologs of biofuel enzymes (metalogs) in different EC Classes and metagenomes.

\begin{tabular}{|c|c|c|c|c|c|c|c|c|}
\hline Metagenome ID & Metagenome Source & EC 1 & EC 2 & EC 3 & EC 4 & EC 5 & EC 6 & Total \\
\hline mgm4440324 & Marine Biome & 52 & 52 & 7 & 20 & 2 & 20 & 153 \\
\hline mgm4440329 & Hypersaline & 118 & 66 & 26 & 20 & 5 & 43 & 278 \\
\hline mgm4441050 & Hypersaline & 25 & 5 & 1 & 5 & 0 & 8 & 44 \\
\hline mgm4441102 & Hydrothermal Vent & 2047 & 1179 & 281 & 866 & 114 & 744 & 5231 \\
\hline mgm4443684 & Freshwater & 1271 & 577 & 383 & 485 & 75 & 603 & 3394 \\
\hline mgm4448052 & Aquatic Biome & 1542 & 831 & 726 & 544 & 40 & 718 & 4401 \\
\hline mgm4449252 & Grassland & 590 & 222 & 285 & 118 & 32 & 186 & 1433 \\
\hline mgm4460449 & Hot Spring & 3992 & 2876 & 878 & 1619 & 214 & 2393 & 11972 \\
\hline mgm4466309 & Coral Reef & 1075 & 629 & 240 & 426 & 53 & 499 & 2922 \\
\hline mgm4467029 & Large Lake & 1569 & 928 & 1373 & 636 & 61 & 445 & 5012 \\
\hline mgm4477803 & Lake & 8874 & 3901 & 1479 & 2595 & 401 & 2715 & 19965 \\
\hline mgm4478241 & $\begin{array}{l}\text { Extreme Aquatic Habitat } \\
\text { (Drilling) }\end{array}$ & 1732 & 849 & 195 & 372 & 10 & 870 & 4028 \\
\hline mgm4479942 & Village Biome & 671 & 238 & 194 & 132 & 29 & 191 & 1455 \\
\hline mgm4487639 & Forest Biome & 694 & 316 & 94 & 169 & 25 & 157 & 1455 \\
\hline mgm4494621 & Activated Sludge & 12812 & 5781 & 1027 & 4268 & 236 & 4758 & 28882 \\
\hline mgm4516289 & Aquatic Biome & 1096 & 585 & 492 & 404 & 62 & 446 & 3085 \\
\hline mgm4523306 & $\begin{array}{l}\text { Anthropogenic Terrestrial } \\
\text { Biome }\end{array}$ & 6 & 3 & 12 & 2 & 0 & 1 & 24 \\
\hline mgm4527699 & Cropland Biome & 3322 & 1869 & 3383 & 1437 & 475 & 850 & 11336 \\
\hline mgm4528623 & Cropland Biome & 627 & 292 & 457 & 194 & 59 & 231 & 1860 \\
\hline mgm4537095 & $\begin{array}{l}\text { Mediterranean Forests, } \\
\text { Woodlands, Shrub }\end{array}$ & 843 & 906 & 37 & 191 & 10 & 642 & 2629 \\
\hline mgm4559623 & Aquatic Biome & 470 & 248 & 210 & 140 & 35 & 182 & 1285 \\
\hline mgm4571849 & Aquatic Biome & 8303 & 5317 & 5008 & 2039 & 1072 & 2786 & 24525 \\
\hline mgm4571867 & Aquatic Biome & 5667 & 3932 & 4404 & 1871 & 907 & 1604 & 18385 \\
\hline Total & & 57398 & 31602 & 21192 & 18553 & 3917 & 21092 & 153754 \\
\hline
\end{tabular}

2 